\title{
Que Fatores Determinam os Níveis de Atividade Física após Programa de Reabilitação Cardíaca?
}

\author{
What Factors Determine the Levels of Physical Activity after Cardiac \\ Rehabilitation Program?
}

\author{
Diogo SOARES ${ }^{1}$, Sofia VIAMONTE¹, Sandra MAGALHÃES ${ }^{1}$, Maria Miguel RIBEIRO ${ }^{1}$, Ana BARREIRA ${ }^{1}$, Preza \\ FERNANDES ${ }^{2}$, Severo TORRES ${ }^{2}$ \\ Acta Med Port 2013 Nov-Dec;26(6):689-698
}

\begin{abstract}
RESUMO
Introdução: Os Programas de Reabilitação Cardíaca ganharam enorme relevância na prevenção de doenças cardiovasculares constituindo um desafio assegurar a prática de exercício físico regular durante e após o fim do programa supervisionado. O objetivo deste trabalho foi determinar os fatores que influenciam os hábitos de atividade física 12 meses após um Programa de Reabilitação Cardíaca.
\end{abstract}

Material e Métodos: Estudo prospetivo abrangendo 580 doentes com cardiopatia isquémica consecutivamente orientados para Programas de Reabilitação Cardíaca na Unidade de Reabilitação Cardiovascular do Centro Hospitalar do Porto, entre Janeiro de 2008 e Junho de 2011. Avaliaram-se os níveis de atividade física através do International Physical Activity Questionnaire realizado no início do programa, aos 3 e 12 meses depois. Foram testados como potenciais determinantes dos hábitos de atividade física a longo prazo: idade; sexo; fatores de risco modificáveis; capacidade funcional (alcançada em prova de esforço); análises laboratoriais (HbA1c, perfil lipídico, Proteína C Reativa e Peptideo Natriurético Cerebral). Realizou-se análise de regressão linear para identificar os preditores significativos e encontrar o melhor ajuste do modelo.

Resultados: A idade avançada, género feminino, a capacidade funcional, níveis de atividade física baixos previamente ao Programa de Reabilitação Cardíaca e uma fraca evolução do International Physical Activity Questionnaire durante o programa foram os melhores preditores univariáveis de uma evolução menos favorável do International Physical Activity Questionnaire nos 12 meses de follow-up. A análise de regressão linear multivariável concluiu que o melhor modelo explicativo incluía: idade, género, evolução do IPAQ no programa $\left(R^{2}\right.$ ajust $\left.=0,318 ; f=60,62 ; p<0,001\right)$.

Conclusão: A identificação de subgrupos de doentes com menor tendência à prática de atividade física permite desenvolver estratégias individualizadas, maximizando o potencial terapêutico e preventivo dos Programas de Reabilitação Cardíaca.

Palavras-chave: Centros de Reabilitação Física; Doença Coronária/reabilitação; Doenças do Coração/reabilitação; Qualidade de Vida; Reabilitação; Terapia por Exercício.

\section{ABSTRACT}

Introduction: The Cardiac Rehabilitation Programs have gained tremendous importance in the prevention of cardiovascular disease and it's a challenge to ensure the practice of regular exercise during and after the supervised program. The aim of this study was to determine the factors that influence the physical activity habits at 12 months after the Cardiac Rehabilitation Program.

Material and Methods: Prospective study, including 580 patients with ischemic heart disease who were consecutively oriented for Cardiac Rehabilitation Program at Cardiovascular Prevention and Rehabilitation Unit of Centro Hospitalar do Porto, between January 2008 and June 2011. Physical activity levels were measured with International Physical Activity Questionnaire which was calculated at the beginning of the program, 3 and 12 months later. The following variables were chosen and tested as potential determinants of physical activity habits of 12 months after program: age; sex; modifiable risk factors; functional capacity (achieved in treadmill stress test); laboratory analysis (HbA1c, lipid profile, C-Reactive Protein and Brain Natriuretic Peptide). A linear regression analysis was carried to identify the significant determinants and to find the best model adjustment.

Results: Advanced age, female gender, functional capacity and low levels of physical activity prior to the Cardiac Rehabilitation Program, as well as a weak evolution of the International Physical Activity Questionnaire during the program were the best univariable predictors of a less favourable evolution of the International Physical Activity Questionnaire during 12 months of follow-up. A multivariable linear regression analysis showed that the best explanatory model included age, gender and evolution of the International Physical Activity Questionnaire during the supervised program $\left(\mathrm{R}^{2} \mathrm{Adj} .=0.318 ; f=60.62, p<0.001\right)$.

Conclusion: The identification of certain subgroups of patients with lower tendency toward physical activity is beneficial to enable timely and individualized strategies to maximize the therapeutic and preventive potential of the Cardiac Rehabilitation Programs.

Keywords: Coronary Disease/rehabilitation; Exercise Therapy; Heart Diseases/rehabilitation; Quality of Life; Rehabilitation; Rehabilitation Centers.

\section{INTRODUÇÃo}

Os Programas de Reabilitação Cardíaca (PRC) têm sido reconhecidos nas últimas décadas como um componente essencial no tratamento das doenças cardiovasculares (DCV) estando atualmente recomendados para todos os doentes com diagnóstico de Síndrome Coronário Agudo (SCA), ${ }^{1,2}$ angina estável, ${ }^{3}$ insuficiência cardíaca, ${ }^{4}$ após revascularização cirúrgica (Coronary Artery Bypass Graft - CABG) ${ }^{5}$ ou percutânea (intervenção cardíaca percutânea - ICP) ${ }^{6}$ e transplante cardíaco. ${ }^{7}$

1. Serviço de Fisiatria. Centro Hospitalar do Porto. Porto. Portugal.

2. Serviço de Cardiologia. Centro Hospitalar do Porto. Porto. Portugal.

Recebido: 02 de Fevereiro de 2013 - Aceite: 14 de Junho de 2013 | Copyright @ Ordem dos Médicos 2013 
Estão descritos na literatura resultados favoráveis em múltiplas variáveis ${ }^{8}$ : aumento da capacidade funcional, ${ }^{9}$ controlo do perfil lipídico e glicémico, ${ }^{10}$ redução do Índice de Massa Corporal (IMC), da pressão arterial e dos parâmetros inflamatórios, ${ }^{10-15}$ melhoria dos fatores psicológicos e da qualidade de vida; ${ }^{16,17}$ redução do número de re-hospitalizações, ${ }^{18}$ da morbilidade e mortalidade associada à patologia cardíaca, ${ }^{19-22}$ comprovando-se ainda um benefício económico para o sistema de saúde. Apesar de todos os benefícios, a referenciação dos doentes para os PRC permanece extremamente baixa, com menos de $3 \%$ dos doentes elegíveis a serem admitidos num PRC em Portugal. ${ }^{23}$

A eficácia dos PRC é determinada, em parte, pela sua organização e dinâmica, em que o doente é incentivado a participar de forma ativa no seu processo de reabilitação, com vista à reinserção precoce na comunidade. A informação fornecida ao doente relativamente à sua patologia, aos fatores de risco cardiovascular (FRCV) e aos objetivos do PRC são igualmente importantes para alcançar o sucesso desejado na melhoria dos perfis de risco, intimamente ligados à morbi-mortalidade.

Um dos grandes desafios para os profissionais envolvidos na Reabilitação Cardíaca é modificar o estilo de vida dos doentes que participam nos PRC, promovendo, entre outras medidas, a prática regular de atividade física, enfatizando a sua importância na prevenção da doença cardiovascular. A adesão à atividade física tem sido um factor associado a redução de 20 a $30 \%$ na mortalidade total em doentes com patologia cardiovascular conhecida. ${ }^{24}$ No entanto, poucos estudos relatam a evolução dos hábitos de atividade física durante o PRC e a sua manutenção a longo prazo após o término da fase de exercício supervisionado. O nível de atividade física semanal pode ser estimado através de vários métodos, entre os quais questionários auto-preenchidos. O International Physical Activity Questionnaire (IPAQ) é um questionário validado para a população portuguesa e visa quantificar (em METs/minuto/semana) a atividade física realizada ao longo de uma semana, nos diversos contextos das atividades diárias (nomeadamente atividades domésticas, laborais, desportivas e recreativas). ${ }^{25}$ Tem sido utilizado no âmbito da Reabilitação Cardíaca, como instrumento de objetivação dos níveis de atividade física realizados pelos doentes em programa.

O objetivo deste estudo foi avaliar quais os fatores determinantes da evolução dos níveis de atividade física ao longo de 12 meses após um PRC.

\section{MATERIAL E MÉTODOS}

Foi realizado um estudo prospetivo abrangendo 580 doentes com cardiopatia isquémica que foram consecutivamente orientados para PRC na Unida- de de Prevenção e de Reabilitação Cardiovascular (UPRCV) do Centro Hospitalar do Porto, entre Janeiro de 2008 e Junho de 2011e num período máximo de 3 meses após o evento que motivou o internamento hospitalar e a referenciação para o programa. Da amostra inicial foram excluídos 195 doentes por abandono do PRC, intercorrências clínicas que conduziram à sua suspensão ou perda de alguns dados no período de follow-up. Foram assim incluídos neste estudo 385 doentes que completaram PRC sem perda de informação relativa aos parâmetros que se pretendiam avaliar.

Foram estabelecidos três momentos de avaliação: no início do PRC, aos 3 e 12 meses depois. $\mathrm{Na}$ avaliação inicial procedeu-se à realização da história clínica e exame físico abrangendo sistema cardiovascular, músculo-esquelético e neurológico e efetuada recolha dos dados clínicos e características socio-demográficas. Nos diferentes momentos de avaliação estipulados foram ainda recolhidos os dados relativos ao perfil de risco, nomeadamente: medição de pressão arterial com recurso a esfigmomanómetro digital de braço (considerada a média de dois valores medidos após período de cinco minutos de repouso); dados antropométricos incluindo Índice de Massa Corporal $(\mathrm{Kg} / \mathrm{m} 2)$, Perímetro Abdominal e Perímetro de Anca medidos com fita métrica extensível em ortostatismo; registo de hábitos tabágicos; registo de valores de glicemia capilar, $\mathrm{HbA} 1 \mathrm{c}$ e do perfil lipídico (obtido após cumprimento de um jejum de 12 horas) incluindo as avaliações do colesterol total (CT), do colesterol das LDL (C-LDL - low density lipoprotein cholesterol), do colesterol das HDL (C-HDL - high density lipoprotein cholesterol) e triglicerídeos (TG); valores de Proteína C Reativa (PCR) e Peptideo Natriurético Cerebral (BNP).

Nos mesmos períodos os doentes realizaram Prova de Esforço (PE) em tapete rolante motorizado, sob medicação habitual, determinando parâmetros de resposta cronotrópica e hemodinâmica. A capacidade funcional máxima foi estimada em METs obtidos na $P E$, calculados através de equações metabólicas do American College of Sports Medicine (ACSM) para marcha em tapete rolante. ${ }^{26}$

Relativamente à avaliação do nível de atividade física, esta foi efetuada, através do IPAQ, medido em METs/minuto/semana e categorizado de acordo com os cut-off de: inferior a $600 \mathrm{METs/minuto/semana}$ para nível de atividade física baixo (sedentarismo); 600-3 $000 \mathrm{METs/minuto/semana} \mathrm{correspondente} \mathrm{a}$ níveis de atividade física moderados; superior a 3 $000 \mathrm{METs} /$ minuto/semana para níveis de atividade física elevados. Este questionário foi aplicado nos já referidos momentos de avaliação sendo o questionário inicial preenchido no segundo dia de internamento hospitalar no Serviço de Cardiologia, reportando-se portanto aos hábitos de atividade física realizada pelo 
doente previamente ao evento cardíaco.

\section{Intervenção}

A organização do PRC nesta Unidade Hospitalar foi implementada de acordo com recomendações da European Society of Cardiology (ESC) $)^{27}$ e da American Association of Cardiovascular and Pulmonary Rehabilitation (AACVPR). ${ }^{28}$ Inclui sessões psicoeducativas em grupo, aconselhamento individualizado sobre estratégias para controlo dos FRCV (incluindo avaliação nutricional) e sessões de exercício físico supervisionado.

Com uma duração total de 6 a 12 semanas (número estipulado de acordo com fatores individuais, socio-laborais e clínicos), estas sessões decorrem com periodicidade bissemanal na UPRCV, com médico Fisiatra e Cardiologista em regime presencial, respeitando as recomendações internacionais no que se refere ao nível de supervisão e monitorização adequado a cada caso. ${ }^{29} \mathrm{~A}$ duração da sessão varia entre 60 a 90 minutos, englobando um protocolo de exercício constituído por: fase de aquecimento, treino aeróbico (exercício em tapete rolante, cicloergómetro de membros inferiores e superiores), treino de força (com recurso a halteres, bolas medicinais e máquina de fortalecimento muscular), arrefecimento/ relaxamento e exercícios de flexibilização miotendinosa. A intensidade de cada modalidade de exercício é determinada individualmente, com base no cálculo de Frequência Cardíaca de Treino (FCT) através do método de Karvonen, ${ }^{26}$ utilizando os dados recolhidos nas provas de esforço; é complementada com a perceção subjetiva do esforço registada na escala de Borg. ${ }^{29}$ Durante as sessões foi efetuada monitorização contínua de ECG por telemetria, complementada pelo uso de cardiofrequencímetros pelos doentes, procedendo-se ao ajuste periódico da intensidade do exercício sempre que indicado (visando os 50 a $80 \%$ da reserva cronotrópica).

Adicionalmente, foi incentivada em todos os doentes a prática do exercício nos restantes dias da semana, com base nos princípios gerais da prescrição, fomentando o seu incremento de forma segura. O plano exercício prescrito previa a realização de 40 a 60 minutos diários de atividade física moderada, com parâmetros similares aos fornecidos nas sessões supervisionadas, nomeadamente a FCT (aferida pelo doente com cardiofrequencímetro) e perceção subjetiva de esforço (11 - 'algo leve' a 13 - 'algo forte' na escala de Borg).

\section{Análise Estatística}

O processamento estatístico dos dados foi efetuado com o programa SPSS versão 19, utilizando testes paramétricos e não paramétricos para amostras emparelhadas e análise de regressão linear univariável e multivariável. A análise de regressão linear teve como variável dependente a diferença do score do IPAQ no início e aos 12 meses. A escolha das variáveis preditoras foi baseada na comparação do melhor ajuste dos vários modelos criados, após tratamento diagnóstico de observações influentes e da confirmação dos pressupostos necessários à análise de regressão linear.

Tabela 1 - Características da amostra na admissão para PRC.

Idade, média (DP)

$60,41 \pm 10,86$

Sexo

- Masculino

$293(76,1 \%)$

- Feminino

Diagnóstico de admissão

- Síndrome coronário agudo

$257(66,8 \%)$

- ICP eletiva

$67(17,4 \%)$

- Angina Pectoris Estável

$20(5,19 \%)$

- CABG

$35(9,1 \%)$

- Insuficiência cardíaca

$6(1,6 \%)$

FRCV

- IMC $\geq 25$

$266(69,1 \%)$

- Dislipidemia

- Hipertensão arterial

$239(62,1 \%)$

- Diabetes Mellitus tipo 2

$133(34,5 \%)$

- Tabagismo

$116(30,1 \%)$

- Stress

$88(22,9 \%)$

Níveis de atividade física semanal (IPAQ)

- Sedentarismo

- Atividade moderada

- Atividade vigorosa 


\section{RESULTADOS}

O grupo de estudo incluiu 385 doentes, a maioria do sexo masculino $(76,1 \%)$, com idades compreendidas entre os 30 e os 84 anos. $66,8 \%$ dos doentes foram admitidos com o diagnóstico de SCA. A caracterização completa da amostra é apresentada na Tabela 1 .

No início do PRC, de acordo com os resultados do IPAQ, 50,4\% dos doentes reportavam um baixo índice de atividade física semanal, sendo os doentes do sexo feminino ou aqueles em que a insuficiência cardíaca constituiu o diagnóstico de admissão os que apresentavam valores mais baixos no IPAQ. Na Fig. 1 apresentam-se os índices de atividade física reportados de acordo com o escalão etário. A Fig. 2 demonstra a capacidade funcional (METs, estimados na PE inicial) verificando-se valores inferiores nos escalões mais avançados.

Ao longo do PRC foi notório o aumento global dos níveis de atividade física semanal $(+204 \% ; p<0,001)$ assim como da capacidade funcional, registada através da PE realizada após o mesmo $(+17 \% ; p<0,001)$ (Fig.s 3 e 4). Os doentes sedentários (com scores inferiores a $600 \mathrm{METs} /$ minuto/semana), os que foram admitidos com diagnóstico de 'Angina Estável' e os que se encontravam na faixa etária dos 60 a 69 anos foram os subgrupos onde a evolução dos resultados do IPAQ foi mais acentuada (Tabelas 2, 3 e 4). Não se registaram diferenças significativas entre os sexos (Tabela 5). Os doentes com idade avançada, sobretudo na faixa etária superior a 80 anos, apresentaram os piores resultados no aumento dos scores do IPAQ.

$\mathrm{Na}$ avaliação aos 12 meses observou-se uma queda dos valores médios do IPAQ comparativamente aos registados após o término do PRC $(-20 \% ; p$ $<0,001)$. Segundo o diagnóstico de admissão, a redução dos valores variou entre os $7 \%$ e os $58 \%$ tendo sido apenas significativa no subgrupo de doentes admitidos após SCA $(-22 \% ; p<0,001)$. Os doentes com idade entre os 60-80 anos apresentaram uma descida significativa do score do IPAQ sendo a diferença mais acentuada no sexo feminino. Apenas os indivíduos que previamente já apresentavam hábitos de atividade física superiores a $3000 \mathrm{METs} /$ minuto/ semana mantiveram os seus hábitos na avaliação aos 12 meses.

A idade avançada, o género feminino, a capacidade funcional e os níveis prévios de atividade físi-

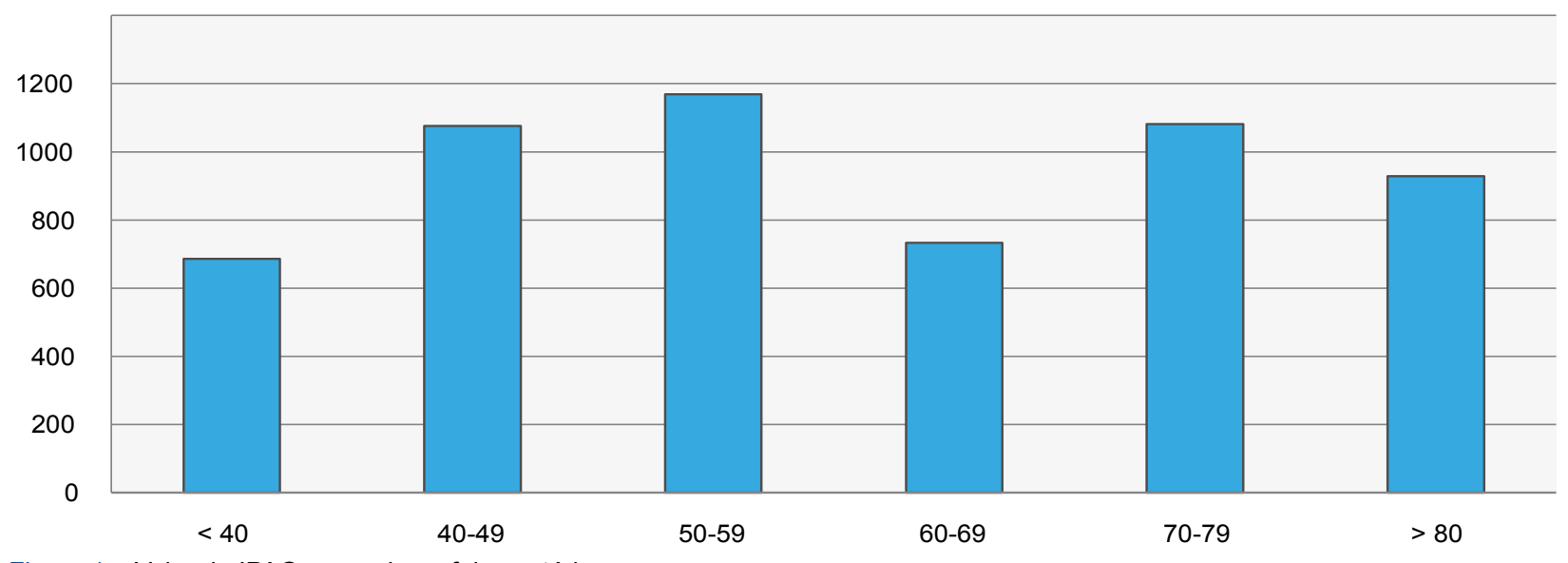

Figura 1 - Valor do IPAQ segundo as faixas etárias

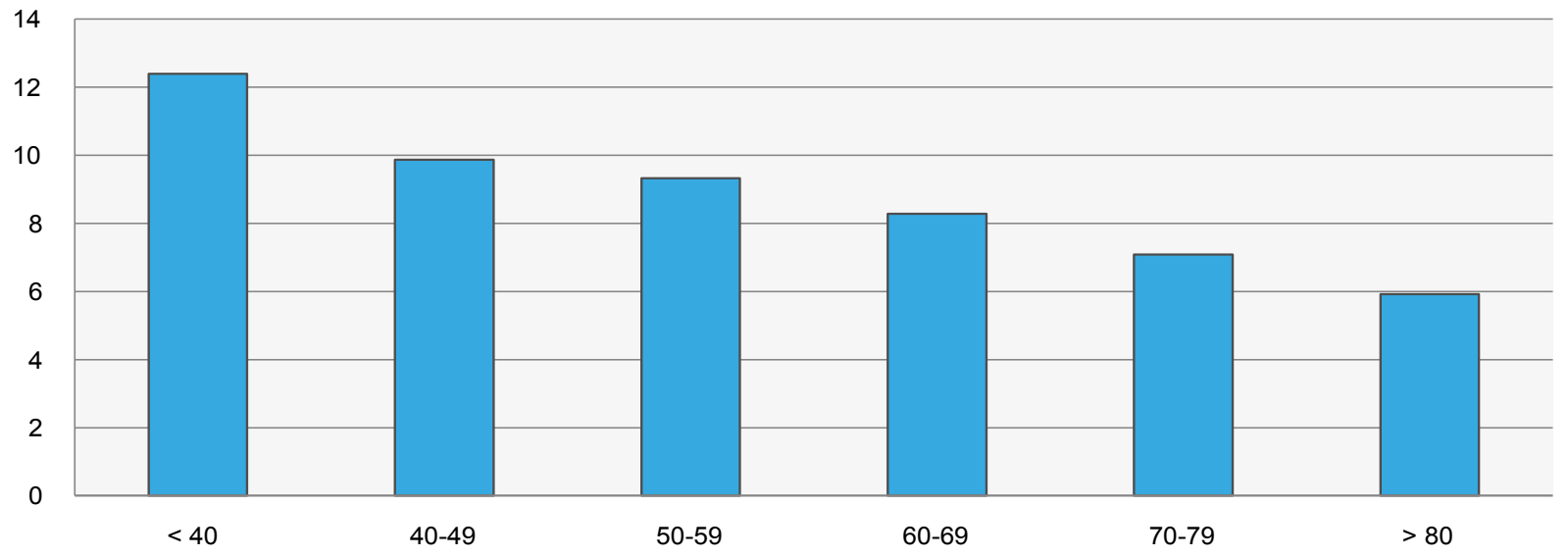

Figura 2 - Capacidade funcional segundo as faixas etárias 
ca baixos previamente ao programa, bem como uma fraca evolução do IPAQ durante o PRC foram os melhores preditores univariáveis de uma evolução menos favorável do IPAQ durante os 12 meses de follow-up (Tabela 6). A análise de regressão linear multivariável permitiu concluir que o melhor modelo explicativo incluía a idade, o género e a evolução do IPAQ no PRC ( $R^{2}$ ajust. $\left.=0,318 ; f=60,62 ; p<0,001\right)$. Todas as outras variáveis incluídas no modelo de regressão linear não revelaram significância estatística.

\section{DISCUSSÃO}

No presente estudo, envolvendo 385 doentes, verificou-se um aumento significativo dos níveis de atividade física semanal após o PRC em todos os doentes, independente da idade, do género ou da patologia de base, sendo que os indivíduos previamente sedentários, do sexo masculino ou com diagnóstico de admissão de angina estável aqueles que apresentaram os melhores resultados. $\mathrm{Na}$ avaliação realizada aos 12 meses ocorreu uma descida ligeira mas significativa do score obtido através do IPAQ, mantendo-se no entanto uma média superior aos valores do início do programa, reforçando o impac- to dos PRC a longo prazo no estilo de vida destes doentes. Através da análise dos vários subgrupos verificamos que a diminuição dos scores do IPAQ aos 12 meses foi mais evidente nos doentes com angina estável e do sexo feminino. A análise de regressão linear permitiu concluir que determinadas características no início do PRC como a idade avançada, o sexo feminino, um valor baixo do IPAQ ou dos METs alcançados na prova de esforço são preditores de uma evolução menos favorável dos níveis de atividade física ao longo do primeiro ano após evento. O modelo de regressão mais ajustado que incluiu as variáveis idade, género e evolução do IPAQ durante o PRC, explica em $31,8 \%$ a variação do IPAQ nos primeiros 12 meses.

Da revisão da literatura efetuada apenas dois estudos semelhantes foram encontrados. Ades et $a^{30}$ verificaram que características na admissão para PRC como a idade avançada, o sexo feminino, uma baixa capacidade funcional, a presença de comorbilidades médicas e de depressão eram fatores preditores de baixos níveis de atividade física iniciais. A evolução da capacidade funcional e do score de depressão durante o PRC foram as variáveis preditoras

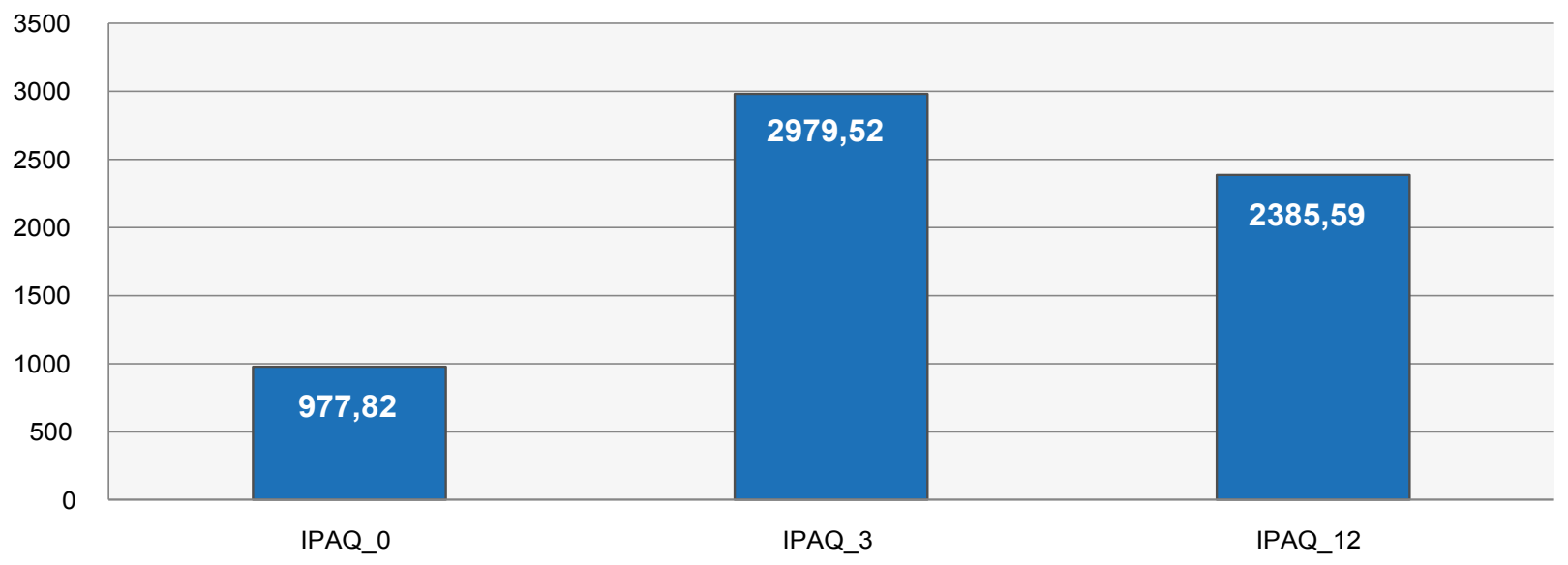

Figura 3 - Evolução do score do IPAQ

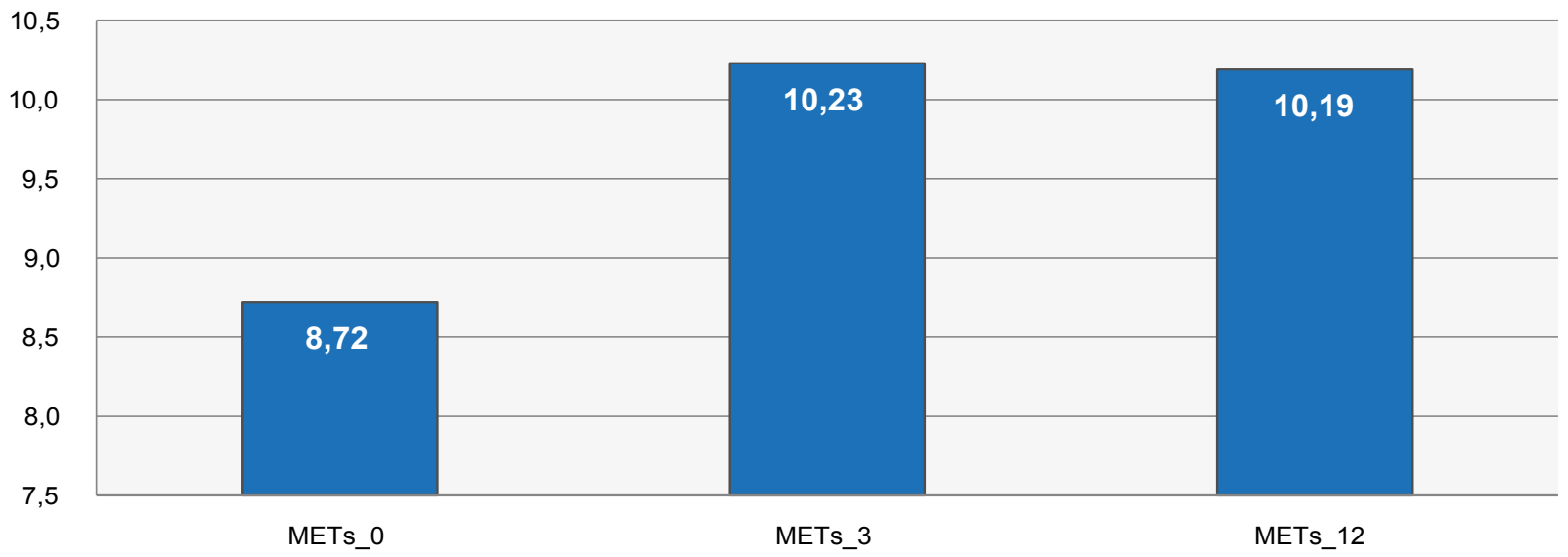

Figura 4 - Evolução da capacidade funcional em METs 
Tabela 2 - Evolução do IPAQ segundo o nível de atividade física à admissão

\begin{tabular}{|c|c|c|c|c|c|c|}
\hline Nível de atividade física & $\mathbf{N}^{\circ}$ Doentes & IPAQ_0 & IPAQ_3 & $\begin{array}{l}\text { Evolução } \\
0 \text {-3meses }\end{array}$ & IPAQ_12 & $\begin{array}{l}\text { Evolução } \\
\text { 3-12meses }\end{array}$ \\
\hline Sedentarismo & 194 & 139,50 & 2547,61 & $+1726 \%(p<0,001)$ & 1878,74 & $-26 \%(p<0,001)$ \\
\hline Atividade moderada & 169 & 1383,14 & 3142,13 & $+127 \%(p<0,001)$ & 2617,46 & $-17 \%(p=0,001)$ \\
\hline Atividade intensa & 22 & 5256,68 & 5539,31 & $+5 \%(p=0,455)$ & 5073,93 & $-8 \%(p=0,528)$ \\
\hline
\end{tabular}

Tabela 3 - Evolução do IPAQ segundo o diagnóstico de admissão

\begin{tabular}{|c|c|c|c|c|c|c|}
\hline Diagnóstico & $\mathbf{N}^{\circ}$ Doentes & IPAQ_0 & IPAQ_3 & $\begin{array}{l}\text { Evolução } \\
0-3 \text { meses }\end{array}$ & IPAQ_12 & $\begin{array}{l}\text { Evolução } \\
\text { 3-12meses }\end{array}$ \\
\hline SCA & 257 & 1094,33 & 3009,24 & $+175 \%(p<0,001)$ & 2360,29 & $-22 \%(p<0,001)$ \\
\hline ICP eletiva & 67 & 817,68 & 3076,87 & $+276 \%(p<0,001)$ & 2651,31 & $-14 \%(p=0,134)$ \\
\hline Angina estável & 20 & 575,40 & 2620,10 & $+355 \%(p<0,001)$ & 1495,13 & $-42 \%(p=0,031)$ \\
\hline CABG & 35 & 767,36 & 3025,54 & $+294 \%(p<0,001)$ & 2732,43 & $-10 \%(p=0,572)$ \\
\hline IC & 6 & 351,00 & 1549,25 & $+341 \%(p=0,043)$ & 1447,00 & $-7 \%(p=0,756)$ \\
\hline
\end{tabular}

SCA - Síndrome Coronário Agudo; ICP - Intervenção Cardíaca Percutânea; CABG - Coronary Artery Bypass Graft; IC - insuficiência cardíaca

Tabela 4 - Evolução do IPAQ segundo as faixas etárias

\begin{tabular}{|c|c|c|c|c|c|c|}
\hline Faixa etária & $\mathbf{N}^{\circ}$ Doentes & IPAQ_0 & IPAQ_3 & $\begin{array}{l}\text { Evolução } \\
0-3 \text { meses }\end{array}$ & IPAQ_12 & $\begin{array}{l}\text { Evolução } \\
\text { 3-12meses }\end{array}$ \\
\hline $0-39$ anos & 12 & 685,88 & 2703,00 & $+294 \%(p=0,003)$ & 2360,29 & $-12 \%(p=0,241)$ \\
\hline $50-59$ anos & 113 & 1168,99 & 3107,39 & $+165 \%(p<0,001)$ & 1638,58 & $-47 \%(p=0,019)$ \\
\hline $60-69$ anos & 125 & 733,28 & 3078,55 & $+319 \%(p<0,001)$ & 2732,43 & $-11 \%(p<0,001)$ \\
\hline $70-79$ anos & 78 & 1081,24 & 2435,51 & $+125 \%(p<0,001)$ & 1447,00 & $-40 \%(p<0,001)$ \\
\hline$\geq 80$ anos & 8 & 928,13 & 1247,63 & $+34 \%(p=0,042)$ & 1228,71 & $-1 \%(p=0,285)$ \\
\hline $\begin{array}{l}\text { da evolução } \\
\text { mesmo. Já S } \\
\text { de idade supe } \\
\text { superior a } 30 \\
\text { os níveis de } \\
\text { rante o períoc }\end{array}$ & $\begin{array}{l}\text { níveis de a } \\
\text { irer et al }{ }^{31} \\
\text { a } 70 \text { anos, } \\
\text { esentavam } \\
\text { dade física } \\
\text { n que se e }\end{array}$ & $\begin{array}{l}\text { ade físi } \\
\text { luíram } \\
\text { exo fem } \\
\text { uldade } \\
\text { ejáveis, } \\
\text { travam }\end{array}$ & $\begin{array}{l}\text { urante o } \\
\text { doentes } \\
\text { ou IMC } \\
\text { atingirem } \\
\text { smo du- } \\
\text { PRC. }\end{array}$ & \multicolumn{3}{|c|}{$\begin{array}{l}\text { A importância atribuída à atividade física ganhou } \\
\text { uma nova dimensão quando em } 1992 \text { a American } \\
\text { Heart Association considerou a inatividade física um } \\
\text { fator de risco importante para a doença cardiovas- } \\
\text { cular. }^{32} \text { Desde então vários estudos exploraram os } \\
\text { potenciais efeitos da atividade física na prevenção }\end{array}$} \\
\hline
\end{tabular}


Tabela 5 - Evolução do IPAQ segundo o género

\begin{tabular}{lcccccc}
\hline Género & $\mathbf{N}^{\circ}$ doentes & IPAQ_0 & IPAQ_3 & $\begin{array}{c}\text { Evolução } \\
\mathbf{0 - 3 m e s e s}\end{array}$ & IPAQ_12 & $\begin{array}{c}\text { Evolução } \\
\mathbf{3 - 1 2 m e s e s}\end{array}$ \\
\hline Masculino & 293 & 1066,36 & 3261,04 & $+205 \%(p<0,001)$ & 2678,57 & $-17 \%(p<0,001)$ \\
Feminino & 92 & 695,84 & 2082,95 & $+199 \%(p<0,001)$ & 1452,52 & $-30 \%(p<0,001)$ \\
\hline
\end{tabular}

Tabela 6 - Determinantes da evolução do IPAQ nos 12 meses após PRC

\begin{tabular}{|c|c|c|c|}
\hline Preditor univariável & Coeficiente de correlação & $\mathbf{R}^{2}$ & $p$ \\
\hline Idade & $-0,214$ & 0,046 & $<0,001$ \\
\hline Sexo & $-0,180$ & 0,033 & $<0,001$ \\
\hline METs_0 & 0,154 & 0,024 & 0,003 \\
\hline IPAQ_0 & 0,338 & 0,114 & $<0,001$ \\
\hline Evolução IPAQ 0-3 & 0,539 & 0,291 & $<0,001$ \\
\hline
\end{tabular}

primária e secundária das doenças cardiovasculares. Está documentada uma relação inversa entre atividade física e doença cardiovascular ${ }^{33}$, mediada pela alteração nos FRCV, particularmente nos fatores inflamatórios/hemostáticos e na pressão arterial ${ }^{34}$. Foi demonstrada a existência de uma relação dose-resposta entre a atividade física e o risco cardiovascular. Uma recente meta-análise efetuada por Sattelmair et $\mathrm{a}^{35}$ verificou que indivíduos com níveis de atividade física que cumpriam com as guidelines americanas para a prática de atividade física apresentavam um risco de doença coronária inferior em $14-20 \%$ ao dos indivíduos sedentários, sendo que níveis de atividade física superiores cursavam com maior redução do risco de DCV. Contudo, a prática de atividade física é igualmente benéfica nos indivíduos que não atingem os níveis exigidos pelas guidelines, sendo no entanto os resultados menos notórios. Relativamente ao risco de re-enfarte, Steffen-Batey et al ${ }^{36}$ concluíram que os doentes previamente ativos que mantiveram os seus níveis de atividade física após um EAM apresentaram um risco de re-enfarte $60 \%$ inferior ao dos doentes que continuaram sedentários. Por outro lado, os doentes que aumentaram os níveis de atividade física apresentaram um risco de re-enfarte $78 \%$ inferior ao dos doentes sedentários. A evolução da capacidade funcional influencia de igual modo o risco de mortalidade, ocorrendo uma diminuição de 8 a $14 \%$ do risco de mortalidade por cada MET alcançado. Da análise destes dados se conclui que o exercício físico é um excelente coadjuvante no tratamento dos doentes com DCV.

A idade avançada está habitualmente associada a múltiplas comorbilidades, nomeadamente do foro musculo-esquelético, neurológico e cardiovascular que originam uma diminuição da capacidade funcional e limitam a prática regular de atividade física. No nosso estudo, no início do PRC a média do IPAQ e dos METs nos doentes com mais de 60 anos era ligeiramente inferior à dos restantes. No entanto, apesar de se observar uma relação inversa entre a idade e a capacidade funcional, não se verificou semelhante tendência entre a idade e os níveis de atividade física (Fig.s 1 e 2). A hipótese mais provável para este facto é que, apesar da população idosa relatar níveis razoáveis de atividade física, esta não é realizada com a intensidade e duração suficientes para promover os mecanismos fisiológicos que se traduzam num aumento significativo da capacidade funcional. Ao longo do PRC a média do valor do IPAQ dos doentes com mais de 60 anos evoluiu significativamente, observando-se posteriormente a sua redução. Curiosamente, o subgrupo de doentes com mais de 80 anos $(n=8)$, para além de ter sofrido um aumento significativo no valor do IPAQ durante o PRC foi aquele onde a redução aos 12 meses foi menor $(-1 \%$; $p=$ $0,285)$. Embora nestas faixas etárias, exista alguma dificuldade em implementar a prática de exercício físico de intensidade moderada-elevada, foi documentada uma diminuição da taxa de mortalidade global e por doença cardiovascular mesmo para baixos níveis de atividade física, o que fundamenta a importância de incluir estes indivíduos mais idosos nos PRC. ${ }^{37-39}$

A capacidade funcional e os níveis de atividade física são tendencialmente inferiores no sexo feminino. ${ }^{40,41}$ Neste estudo, apesar de o incremento no IPAQ ter sido semelhante em ambos os sexos, aos 12 meses observou-se no sexo feminino uma redu- 
ção em $30 \%$ dos níveis de alcançados no final do PRC, comparado com $17 \%$ no sexo masculino. Alguns fatores psicológicos e sociais poderão justificar a diferença encontrada entre géneros. $O$ sexo feminino terá uma maior tendência à aceitação da doença como uma situação inexorável. Ultrapassada a fase aguda e subaguda após o evento cardíaco, a mulher frequentemente retoma a sua atividade profissional e reassume o seu papel no agregado familiar, o que pode reduzir o tempo disponível para a prática de atividade física. Sabendo que o papel do exercício como agente de prevenção primária é ligeiramente superior no sexo feminino este facto alerta para a necessidade de desenvolver estratégias que visem otimizar a adesão a longo prazo da atividade física nas mulheres.

Segundo os dados de 2011 da Organização Mundial de Saúde, cerca de $54 \%$ da população portuguesa não pratica regularmente exercício. ${ }^{42}$ À entrada no $\mathrm{PRC}$, a percentagem de doentes sedentários era de $50,4 \%$, valor aproximado ao observado na população em geral. Um especial cuidado na prescrição de exercício deverá ser salvaguardado nos doentes previamente sedentários, pela renitência que pode surgir nas fases iniciais do PRC em aderirem a uma prática regular de atividade física. É essencial individualizar a prescrição, ajustando a intensidade, a duração e o tipo de modalidade aos gostos e capacidade funcional do doente.

Foi evidente o papel do PRC na mudança dos hábitos semanais de atividade física nos doentes previamente sedentários em que constatamos um aumento de $1726 \%$ do valor do IPAQ ao fim dos três meses de PRC. Contudo, os ganhos obtidos durante este período reduzem-se significativamente a longo prazo $(-26 \% ; p<0,001)$. Assim, este subgrupo de doentes apresenta um enorme potencial, em que seguramente o efeito terapêutico e preventivo do exercício será mais evidente. Por este motivo, após o término do PRC, estes doentes deverão ser alertados para os riscos da inatividade e incentivados à prática regular de exercício, na medida em que existe a tendência de progressivamente reduzirem os níveis de atividade física, o que contribui para um aumento do seu risco cardiovascular. Os doentes já com níveis de atividade física elevados no início do PRC, aumentaram ainda mais estes níveis durante o programa supervisionado, verificando-se a manutenção dos valores do IPAQ ao longo dos 12 meses (-8\%). Lavie et al ${ }^{43}$ demonstraram que mesmo os doentes com elevada capacidade funcional inicial beneficiavam com o PRC, melhorando o seu perfil lipídico e a sua capacidade funcional.

Na prática clínica verifica-se frequentemente uma relutância por parte dos doentes após um evento cardíaco major em iniciarem a prática de exercício físico de forma regular e intensidade suficiente para atingir os efeitos pretendidos. Instala-se um sentimento de receio, associado ao mito de que o exercício físico é um agente deletério para os indivíduos portadores de doença cardíaca. Os PRC procuram sensibilizar os doentes para os benefícios da prática de exercício físico em fases precoces da sua recuperação. A supervisão, a monitorização e o acompanhamento médico nas classes de exercício permitem simultaneamente a aquisição de uma progressiva sensação de segurança e confiança à medida que a intensidade do exercício vai sendo aumentada. A utilização de sistemas de automonitorização, como por exemplo os cardiofrequencímetros, poderá funcionar não só como um elemento que confere segurança ao doente na prática de exercício mas também como um elemento catalisador para a sua prática. A marcha, dada a sua segurança, acessibilidade, baixo risco de lesões e comprovados efeitos na redução da taxa de mortalidade em doentes com doença cardiovascular, é habitualmente o tipo de exercício prescrito nos doentes após um evento cardíaco, especialmente naqueles em que os níveis prévios de atividade física eram reduzidos e exibiam uma baixa capacidade funcional. ${ }^{44}$

A continuidade de um programa de exercício regular instituído durante o PRC, que cumpra com as guidelines/recomendações propostas, é usualmente difícil de ser assegurada. Com o recurso a acelerómetros verificou-se que apenas cerca de $50 \%$ dos doentes após PRC aderiam à prática de exercício com a intensidade recomendada pelo médico e que a os níveis de atividade física adequados eram apenas alcançados nos dias de PRC e não nos restantes dias da semana. ${ }^{45,46}$ Contudo, encontramos na literatura registo a avaliações aos 2 e 6 anos após PRC onde está descrita a manutenção dos padrões da atividade física alcançados no PRC. ${ }^{47,48}$ Esta variedade é devida em parte à variedade de formas utilizadas para o cálculo dos níveis de atividade física (acelerómetros, podómetros, questionários) e por outro à falta de diferenciação entre atividade física global e atividade física de lazer. O efeito protetor do exercício parece ser mais notório quando a atividade física é realizada como atividade de lazer do que quando é realizada como atividade ocupacional/laboral. ${ }^{49-51}$ Tal diferença pode ser explicada pelo facto de as atividades serem predominantemente do tipo anaeróbico com menor repercussão cardiovascular.

Permanece a discussão sobre qual será o fator mais determinante na prescrição de exercício, se a intensidade, se a duração do exercício. Tanasescu et $a^{52}$ concluíram que quer a intensidade, quer a duração do exercício estavam ambos inversamente associados ao risco de doença coronária. Contudo a intensidade evidenciou um efeito mais significativo na incidência da doença coronária. Já Manson et $a^{53}$ verificaram que para o mesmo volume de exercício 
alcançado, a redução do risco cardiovascular era substancialmente maior para exercícios de duração mais prolongada. Estudos mais recentes afirmam que a prática de atividade física de intensidade moderada-intensa em curtos períodos de tempo $1<10$ min) parece influenciar favoravelmente o risco cardiovascular. ${ }^{54}$

No presente estudo, a avaliação dos níveis semanais de atividade física foi efetuada recorrendo ao IPAQ, um inquérito já validado em Português para tal efeito. Contudo, visto tratar-se de um questionário, apresenta como grande desvantagem o facto de ser uma forma subjetiva de aferir os níveis de atividade física, em que o doente relata o tipo, a duração e a frequência com que praticou determinada atividade. Outros métodos de avaliação, que confiram maior objetividade, nomeadamente os acelerómetros, poderão ser futuramente utilizados como complemento dos diversos questionários existentes.

\section{Limitações do estudo}

O objetivo deste estudo consistiu em descrever a evolução dos níveis de atividade física dos diferentes subgrupos e avaliar quais os fatores determinantes da evolução global do IPAQ ao longo do $1^{\circ}$ ano. Não foi efetuada análise estatística com o intuito de determinar os vários fatores preditores do IPAQ para cada subgrupo, como por exemplo para a idade, o género ou o diagnóstico de admissão. Estudos futuros poderão estabelecer uma relação entre a evolução dos níveis de atividade física e o impacto nos vários fatores de risco cardiovasculares em doentes com doença cardiovascular conhecida.

O facto de se tratar de um estudo que incluiu apenas os doentes que cumpriram na totalidade o PRC,

\section{REFERÊNCIAS}

1. Anderson JL, Adams CD, Antman EM, Bridges CR, Califf RM, Casey DE $\mathrm{Jr}$, et al. ACCF/AHA Focused Update Incorporated Into the ACC/AHA 2007 Guidelines for the Management of Patients With Unstable Angina/ Non-ST-Elevation Myocardial Infarction: a report of the American College of Cardiology Foundation/American Heart Association Task Force on Practice Guidelines. Circulation. 2011;123:e426-579.

2. Krumholz HM, Anderson JL, Bachelder BL, Fesmire FM, Finn SD, Foody JM, et al. ACC/AHA Performance measures for adults with ST-elevation and non-ST-elevation myocardial infarction: a report of the American College of Cardiology/American Heart Association Task Force on Performance Measures (Writing Committee to develop performance measures for ST-elevation and non-ST-elevation myocardial infarction): developed in collaboration with the American Academy of Family Physicians and the American College of Emergency Physicians: endorsed by the American Association of Cardiovascular and Pulmonary Rehabilitation, Society for Cardiovascular Angiography and Interventions, and Society of Hospital Medicine. Circulation. 2008;118:2596-648.

3. Fraker TD Jr, Finn SD, Gibbons RJ, Abrams J, Chatterjee K, Daley J, et al. Chronic angina focused update of the ACC/AHA 2002 Guidelines for the management of patients with chronic stable angina: a report of the American College of Cardiology/American Heart Association Task Force on Practice Guidelines Writing Group to develop the focused update of the 2002 Guidelines for the management of patients with chronic stable angina. Circulation. 2007; 116:2762-72.

4. Hunt SA, Abraham WT, Chin MH, Feldman AM, Francis GS, Ganiats TG, et al. Focused update incorporated into the ACC/AHA2005 Guidelines for sendo estes presumivelmente os indivíduos mais recetivos ao cumprimento das indicações dados pelos profissionais de Saúde, enviesa os resultados obtidos, na medida em que grande parte dos doentes que abandonaram o PRC não alcançaram os níveis de atividade física desejados. Seria interessante avaliar o comportamento em termos de atividade física dos doentes que não terminaram o PRC.

\section{CONCLUSÃO}

Neste estudo foram encontrados como fatores preditores da evolução do IPAQ durante o primeiro ano pós-evento cardíaco a idade, o género e a evolução do IPAQ durante o PRC. O aumento dos níveis de atividade física e a sua manutenção ao longo do tempo é um dos principais desafios para os profissionais que participam nos PRC. Estes programas assumem uma enorme relevância na educação e no incentivo à prática regular de exercício físico. A identificação de determinados subgrupos de doentes menos responsivos poderá ser benéfica ao permitir desenvolver estratégias atempadas e individualizadas maximizando o potencial terapêutico e preventivo do PRC. Futuras investigações deverão procurar identificar outras variáveis que condicionem os níveis de atividade física neste tipo de população.

\section{CONFLITOS DE INTERESSE}

Os autores declaram a inexistência de conflitos de interesse.

\section{FONTES DE FINANCIAMENTO}

Os autores declaram que não houve fontes de financiamento.

the Diagnosis and Management of Heart Failure in Adults: a report of the American College of Cardiology Foundation/American Heart Association Task Force on Practice Guidelines: developed in collaboration with the International Society for Heart and Lung Transplantation. Circulation. 2009;119:e391-479.

5. Hillis LD, Smith PK, Anderson JL, Bittl JA, Bridges CR, Byrne JG, et al. 2011 ACCF/AHA Guideline for Coronary Artery Bypass Graft Surgery: a report of the American College of Cardiology Foundation/American Heart Association Task Force on Practice Guidelines. Circulation. 2011;124:e652-735.

6. Levine GN, Bates ER, Blankenship JC, Bailey SR, Bittl JA, Cercek $B$, et al. ACCF/AHA/SCAI Guideline for Percutaneous Coronary Intervention: a report of the American College of Cardiology Foundation/ American Heart Association Task Force on Practice Guidelines and the Society for Cardiovascular Angiography and Interventions. Circulation. 2011;124:e574-651.

7. Writing Committee Members, Thomas RJ, King M, Lui K, Oldridge N, Piña IL, et al. AACVPR/ACCF/AHA 2010 update: performance measures on cardiac rehabilitation for referral to cardiac rehabilitation/ secondary prevention services: a report of the American Association of Cardiovascular and Pulmonary Rehabilitation and the American College of Cardiology Foundation/American Heart Association Task Force on Performance Measures (Writing Committee to Develop Clinical Performance Measures for Cardiac Rehabilitation). Circulation. 2010;122:1342-50.

8. Lavie CJ, Thomas RJ, Squires RW, Allison TG, Milani RV. Exercise training and cardiac rehabilitation in primary and secondary prevention 
of coronary heart disease. Mayo Clin Proc. 2009;84:373-83.

9. Stewart KJ, Badenhop D, Brubaker PH, Keteyian SJ, King M. Cardiac rehabilitation following percutaneous revascularization, heart transplant, heart valve surgery, and for chronic heart failure. Chest. 2003;123:210411.

10. Pérez IP, Zapata MA, Cervantes CE, Jarabo RM, Grande C, Plaza R, et al. Cardiac rehabilitation programs improve metabolic parameters in patients with the metabolic syndrome and coronary heart disease. J Clin Hypertens. 2010;12:374-9.

11. Ades PA, Savage PD, Harvey-Berino J. The treatment of obesity in cardiac rehabilitation. J Cardiopulm Rehabil Prev. 2010;30:289-98.

12. Ades PA, Savage PD, Toth MJ, Harvey-Berino J, Schneider DJ, Bunn JY, et al. High-calorie-expenditure exercise: a new approach to cardiac rehabilitation for overweight coronary patients. Circulation 2009;119:2671-8.

13. Lavie CJ, Church TS, Milani RV, Earnest CP. Impact of physical activity, cardiorespiratory fitness, and exercise training on markers of inflammation. J Cardiopulm Rehabil Prev. 2011;31:137-45.

14. Milani RV, Lavie CJ, Mehra MR. Reduction in C-reactive protein through cardiac rehabilitation and exercise training. J Am Coll Cardiol. 2004;43:1056-61.

15. Lavie CJ, Morshedi-Meibodi A, Milani RV. Impact of cardiac rehabilitation on coronary risk factors, inflammation, and the metabolic syndrome in obese coronary patients. J Cardiometab Syndr. 2008;3:136-40.

16. Milani RV, Lavie CJ, Cassidy MM. Effects of cardiac rehabilitation and exercise training program on depression in patients after major coronary events. Am Heart J. 1996;132:726-32.

17. Milani RV, Lavie CJ. Impact of cardiac rehabilitation on depression and its associated mortality. Am J Med. 2007;120:799-806.

18. Ades PA. Cardiac rehabilitation and secondary prevention of coronary heart disease. N Engl J Med. 2001;345:892-902.

19. Heran BS, Chen JM, Ebrahim S, Moxham T, Oldridge N, Rees K, et al. Exercise-based cardiac rehabilitation for coronary heart disease. Cochrane Database Syst Rev. 2011:CD001800.

20. Leizoroviez A, Saint-Pierre A, Vasselon C, Boissel JP. Comparison of a rehabilitation programme, a counselling programme and usual care after an acute myocardial infarction: results of a long-term randomized trial. P.RE.COR.Group. Eur Heart J. 1991;12:612-6.

21. Hammill BG, Curtis LH, Schulman KA, Whellan DJ. Relationship between cardiac rehabilitation and long-term risks of death and myocardial infarction among elderly Medicare beneficiaries. Circulation. 2010;121:63-70.

22. Lawler PR, Filion KB, Eisenberg MJ. Efficacy of exercise-based cardiac rehabilitation post-myocardial infarction: a systematic review and metaanalysis of randomized controlled trials. Am Heart J. 2011;162:571-84. e2.

23. Teixeira M, Sampaio F, Brizida L, Mendes M. Reabilitação Cardíaca em Portugal - Evolução entre 1998 e 2004. Rev Port Cardiol. 2007;27:81525.

24. Hambrecht R, Walther C, Möbius-Winkler S, Gielen S, Linke A, Conradi $\mathrm{K}$, et al. Percutaneous coronary angioplasty compared with exercise training in patients with stable coronary artery disease: a randomized trial. Circulation. 2004;109:1371-8.

25. Craig $C L$, Marshall $A L$, Sjöström M, Bauman $A E$, Booth $M L$, Ainsworth $\mathrm{BE}$, et al. International physical activity questionnaire: 12-country reliability and validity. Med Sci Sports Exerc. 2003;35:1381-95.

26. ACSM's Guidelines for Exercise Testing and Prescription by American College of Sports Medicine. $8^{\text {th }}$ ed. Lippincott Williams \& Wilkins. 2009.

27. Piepoli MF, Corrà U, Benzer W, Bjarnason-Wehrens B, Dendale P, Gaita $D$, et al. Cardiac Rehabilitation Section of the European Association of Cardiovascular Prevention and Rehabilitation. Secondary prevention through cardiac rehabilitation: from knowledge to implementation. A position paper from the Cardiac Rehabilitation Section of the European Association of Cardiovascular Prevention and Rehabilitation. Eur $J$ Cardiovasc Prev Rehabil. 2010;17:1-17.

28. Thomas RJ, King M, Lui K, Oldridge N, Piña IL, Spertus J, et al. AACVPR/ ACC/AHA 2007 performance measures on cardiac rehabilitation for referral to and delivery of cardiac rehabilitation/secondary prevention services. Circulation. 2007;116:1611-42.

29. American Association of Cardiovascular and Pulmonary Rehabilitation. Guidelines for Cardiac Rehabilitation and Secondary Prevention Programs. 4th ed. Champaign: Human Kinetics; 2004.

30. Ades P, Maloney A, Savage P, Carhart R. Determinants of Physical Functioning in Coronary Patients. Arch Intern Med. 1999;159:2357-60.

31. Schairer JR, Keteyian SJ, Ehrman JK, Brawner CA, Berkebile ND. Leisure time physical activity of patients in maintenance cardiac rehabilitation. J Cardiopulm Rehabil. 2003;23:260-5

32. Fletcher GF, Blair SN, Blumenthal J, Caspersen C, Chaitman B, Epstein $S$, et al. Statement on exercise. Benefits and recommendations for physical activity programs for all Americans. A statement for health professionals by the Committee on Exercise and Cardiac Rehabilitation of the Council on Clinical Cardiology, American Heart association. Circulation. 1992;86:340-4.

33. Kokkinos $P$, Myers J. Exercise and physical activity: clinical outcomes and applications. Circulation. 2010;122:1637-48.

34. Mora S, Cook N, Buring JE, Ridker PM, Lee IM. Physical activity and reduced risk of cardiovascular events: potential mediating mechanisms. Circulation. 2007:116:2110-8.

35. Sattelmair J, Pertman J, Ding EL, Kohl HW 3rd, Haskell W, Lee IM. Dose response between physical activity and risk of coronary heart disease: a meta-analysis. Circulation. 2011;124:789-95.

36. Steffen-Batey L, Nichaman MZ, Goff DC Jr, Frankowski RF, Hanis CL, Ramsey DJ. Change in level of physical activity and risk of all-cause mortality or reinfarction: The Corpus Christi Heart Project. Circulation. 2000;102:2204-9.

37. Suaya JA, Stason WB, Ades PA, Normand SL, Shepard DS. Cardiac rehabilitation and survival in older coronary patients. J Am Coll Cardiol. 2009;54:25-33.

38. Hammill BG, Curtis LH, Schulman KA, Whellan DJ. Relationship between cardiac rehabilitation and long-term risks of death and myocardial infarction among elderly Medicare beneficiaries. Circulation. 2010;121:63-70

39. Lee IM, Sesso HD, Oguma Y, Paffenbarger RS Jr. Relative intensity of physical activity and risk of coronary heart disease. Circulation. 2003;107:1110-6.

40. Centers for Disease Control and Prevention. Prevalence of regular physical activity among adults - United States, 2001 and 2005. MMWR Morb Mortal Wkly Rep. 2007;56:1209-12.

41. Troiano RP, Berrigan D, Dodd KW, Mâsse LC, Tilert T, McDowell M Physical activity in the United States measured by accelerometer. Med Sci Sports Exerc. 2008;40:181-8.

42. World Health Organization. Noncommunicable Diseases Country Profiles. Geneve: WHO; 2011

43. Lavie CJ, Milani RV. Patients with high baseline exercise capacity benefit from cardiac rehabilitation and exercise training programs. Am Heart J. 1994;128:1105-9.

44. Murtagh EM, Murphy $\mathrm{MH}$, Boone-Heinonen J. Walking: the first steps in cardiovascular disease prevention. Curr Opin Cardiol. 2010;25:490-6

45. Guiraud T, Granger R, Gremeaux V, Bousquet M, Richard L, Soukarie $\mathrm{L}$, et al. Accelerometer as a tool to assess sedentarity and adherence to physical activity recommendations after cardiac rehabilitation program. Ann Phys Rehabil Med. 2012;55:312-21.

46. Ayabe M, Brubaker PH, Dobrosielski D, Miller HS, Ishi K, Yahiro T, et al. The physical activity patterns of cardiac rehabilitation program participants. J Cardiopulm Rehabil. 2004;24:80-6.

47. Boesch C, Myers J, Habersaat A, Ilarraza H, Kottman W, Dubach P. Maintenance of exercise capacity and physical activity patterns 2 years after cardiac rehabilitation. J Cardiopulm Rehabil. 2005;25:14-21.

48. Mueller L, Myers J, Kottman W, Oswald U, Boesch C, Arbrol N. Exercise capacity, physical activity patterns and outcomes six years after cardiac rehabilitation in patients with heart failure. Clin Rehabil. 2007;21:923-31.

49. Fransson E, De Faire U, Ahlbom A, Reuterwall C, Hallqvist J, Alfredsson L. The risk of acute myocardial infarction: interactions of types of physical activity. Epidemiology. 2004;15:573-82

50. Wennberg $P$, Lindahl B, Hallmans $G$, Messner T, Weinehall L, Johansson $L$, et al. The effects of commuting activity and occupational and leisure time physical activity on risk of myocardial infarction. Eur J Cardiovasc Prev Rehabil. 2006;13:924-30.

51. Besson $\mathrm{H}$, Ekelund $\mathrm{U}$, Brage $\mathrm{S}$, Luben $\mathrm{R}$, Bingham $\mathrm{S}$, Khaw KT. Relationship between subdomains of total physical activity and mortality. Med Sci Sports Exerc. 2008;40:1909-15.

52. Tanasescu M, Leitzmann MF, Rimm EB, Willett WC, Stampfer MJ, Hu FB. Exercise type and intensity in relation to coronary heart disease in men. JAMA. 2002;288:1994-2000.

53. Manson JE, Greenland $P$, LaCroix AZ, Stefanick ML, Mouton CP Oberman A, et al. Walking compared with vigorous exercise for the prevention of cardiovascular events in women. $\mathrm{N}$ Engl $\mathrm{J}$ Med. 2002;347:716-25.

54. Glazer NL, Lyass A, Esliger DW, Blease SJ, Freedson PS, Massaro JM, et al. Sustained and Shorter Bouts of Physical Activity are Related to Cardiovascular Health. Med Sci Sports Exerc. 2013;45:109-15. 
Diogo SOARES, Sofia VIAMONTE, Sandra MAGALHÃES, Maria Miguel RIBEIRO, Ana BARREIRA, Preza FERNANDES, Severo TORRES

\section{Que Fatores Determinam os Níveis de Atividade Física após Programa de Reabilitação Cardíaca?}

Publicado pela Acta Médica Portuguesa, a Revista Científica da Ordem dos Médicos

Av. Almirante Gago Coutinho, 151

1749-084 Lisboa, Portugal.

Tel: +351218428215

E-mail: submissao@actamedicaportuguesa.com

www.actamedicaportuguesa.com

ISSN:0870-399X | e-ISSN: 1646-0758

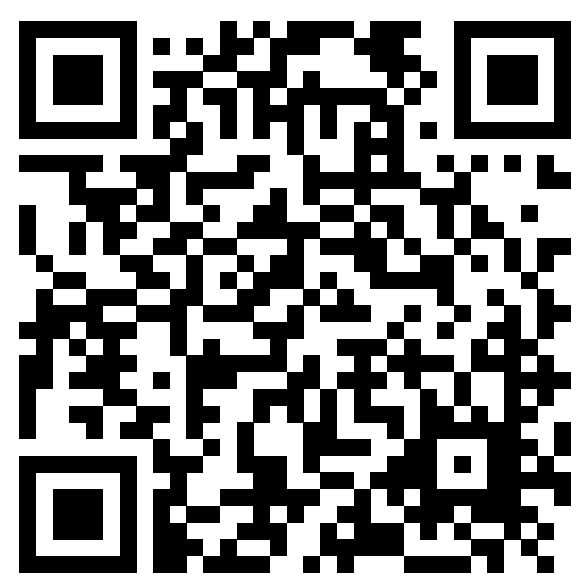

